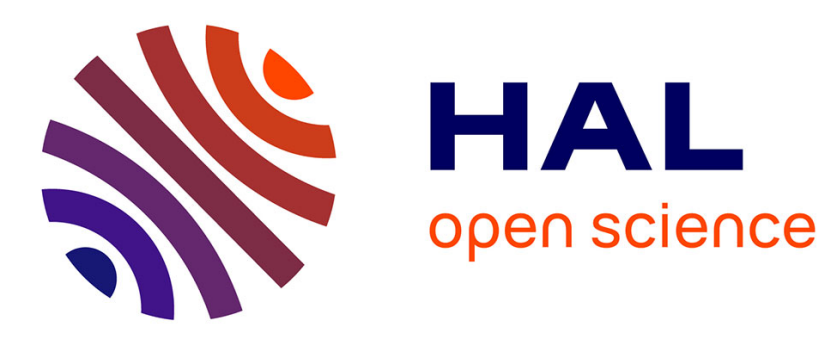

\title{
Slow-light spatial solitons
}

Gil Fanjoux, Jeremy Michaud, Hervé Maillotte, Thibaut Sylvestre

\section{To cite this version:}

Gil Fanjoux, Jeremy Michaud, Hervé Maillotte, Thibaut Sylvestre. Slow-light spatial solitons. Physical Review Letters, 2008, 100 (1), pp.013908. 10.1103/PhysRevLett.100.013908 . hal-00268746

\section{HAL Id: hal-00268746 https://hal.science/hal-00268746}

Submitted on 29 Aug 2013

HAL is a multi-disciplinary open access archive for the deposit and dissemination of scientific research documents, whether they are published or not. The documents may come from teaching and research institutions in France or abroad, or from public or private research centers.
L'archive ouverte pluridisciplinaire HAL, est destinée au dépôt et à la diffusion de documents scientifiques de niveau recherche, publiés ou non, émanant des établissements d'enseignement et de recherche français ou étrangers, des laboratoires publics ou privés. 


\title{
Slow-Light Spatial Solitons
}

\author{
Gil Fanjoux, Jérémy Michaud, Hervé Maillotte, and Thibaut Sylvestre \\ Institut FEMTO-ST Département d'Optique PM Duffieux UMR CNRS-Université de Franche-Comté n ${ }^{\circ}$ 6174, \\ Route de Gray 25030 Besançon Cedex, France
}

(Received 30 July 2007; published 11 January 2008)

\begin{abstract}
We numerically and experimentally report the observation of slow-light spatial solitons in a Kerr medium owing to light amplification by stimulated Raman scattering. This was achieved in a $\mathrm{CS}_{2}$ nonlinear planar waveguide that possesses both a strong self-focusing nonlinearity to generate the spatial Raman soliton and a Raman susceptibility sharp enough to induce the slow-light process simultaneously. We show that the Raman Stokes component is optically delayed by more than 120 ps for a 140 ps Raman pulse duration and only $3 \mathrm{~cm}$ of propagation length, while propagating as a spatial soliton beam.
\end{abstract}

DOI: $10.1103 /$ PhysRevLett. 100.013908

PACS numbers: 42.65.Tg, 42.65.Dr

Slow light has attracted a great deal of attention recently because of many motivations, ranging from fundamental interests to applications in quantum information such as quantum memories, or to optical information management by a controllable time delay between data packets (network buffering, data synchronization) [1]. The main physical origin that leads to the slow or fast light relies on the control of the group velocity of a signal by inducing a strong material dispersion in the medium in the vicinity of the frequency of the signal. This control can be achieved by different means and in different materials.

In a resonant optical material, the change of the group index near a resonance leads to the strong decrease of the group velocity which is intimately linked to a strong absorption. To remove this detrimental absorption, efficient methods for generating a very narrow hole in the absorption line have been performed by using quantum coherence properties of atomic media, such as electromagnetically induced transparency (EIT) in ultracold atomic gases [2], hot gases [3], or very cold solids [4], or by coherent population oscillations $(\mathrm{CPO})$ in solid-state materials at room temperature [5].

In a medium with far-off material resonances, slow and superluminal light can be achieved through a strong groupvelocity dispersion induced by stimulated scattering [610] or by parametric four-wave mixing [11]. The slowing down of the light is closely related to the sharpness of the dispersion curve resulting from the laser-induced resonance. Up to now, most of the work has been performed about slow-light pulses propagating in optical fibers because of their straightforward implementation and potential application. The slow-light process is mainly based on the stimulated Brillouin scattering (SBS) owing to the narrow-gain spectral linewidth (a few MHz) [6,7]. In contrast, stimulated Raman scattering (SRS) in an optical fiber only allows for the achievement of a weaker optical delay because of the larger spectral width of the Raman susceptibility (a few THz) $[8,9]$. Nevertheless, both SRS and SBS processes enable the fine control of the group velocity of a signal pulse by only tuning the resonance at the desired wavelength. In the same way, experiments in a $\mathrm{Ba}\left(\mathrm{NO}_{3}\right)_{2}$ solid-state Raman amplifier with a GHz bandwidth have also resulted in comparable optical delays [10].

Another well-known way to slow down the light is to take advantage of the waveguide dispersion properties of micro- or nanostructured materials, such as photonic crystals, that exhibit a zero group-velocity point near the edge of the photonic band gap [12-14].

In addition, the concept of slow-light solitons is of great interest in order to control not only the group velocity of the light but also its spatiotemporal localization. However, all previous studies mainly concerned temporal solitons [15-21]. As an example, slow-light temporal solitons have been predicted in atomic vapors and Bose-Einstein condensates by Rybin et al. [16]. Another recent theoretical work predicts slow-light soliton generation by evanescent fields in a two-level medium coupled to an external cavity mode when the driving frequency is closed to the band gap edge [17]. Otherwise, Bragg solitons can propagate through the grating at velocities below the speed of light in glass without dispersive broadening, as theoretically $[18,19]$ and experimentally $[20,21]$ demonstrated. Slowlight optical bullets (with a spatiotemporal confinement of the pulse) have also been predicted in an array of nonlinear Bragg-grating waveguides [22].

In this Letter, we introduce in a similar way the new concept of slow-light spatial solitons or spatial solitary waves. To this end, we demonstrate both theoretically and experimentally the simultaneous amplification and slowing down of a spatial Raman soliton in a Kerr medium. The experimental observation is made possible thanks to the use of a $\mathrm{CS}_{2}$ nonlinear liquid-filled slab planar waveguide that exhibits a sufficiently narrow Raman gain bandwidth to generate a strong group-velocity change at Raman frequencies while simultaneously having a strong selffocusing nonlinearity for supporting one-dimensional spatial soliton. In addition to the fundamental interest, slowlight spatial solitons offer the strong advantage to counteract diffraction, which generally limits the propagation length to approximately the Rayleigh range, without the 
requirement of an optical fiber [23]. We demonstrate a simple and efficient technique to obtain slow light, i.e., only a $3-\mathrm{cm}$ propagation in a planar waveguide compared with several hundred meters in optical fibers, a fractional delay (delay on pulse duration ratio) comparable with those previously obtained in SRS or SBS fiber or crystal amplifiers $[6-8,10]$, and on top of that spatial self-confinement.

To illustrate the slow-light effect on a spatial soliton, we have developed a $(2+1) D$ spatiotemporal numerical model based on an extended nonlinear Schrödinger equation including diffraction in the free transverse dimension $x$ of the planar waveguide, linear dispersion, the Kerr nonlinearity, and the delayed Raman response [24]. In the time reference frame of the propagating field, it reads as

$$
\begin{aligned}
\frac{\partial A}{\partial z} & -\frac{i}{2 \beta} \frac{\partial^{2} A}{\partial x^{2}}+\frac{i}{2} \beta_{2} \frac{\partial^{2} A}{\partial t^{2}} \\
& =i \gamma|A|^{2} A+i \gamma A \int_{0}^{\infty} R_{R}(t)\left|A\left(z ; x, t-t^{\prime}\right)\right|^{2} d t^{\prime},
\end{aligned}
$$

where $A(z ; x, t)$ corresponds to the slowly varying envelope of the total electric field propagating in the $z$ direction, $\beta$ is the wave vector, $\beta_{2}$ the group-velocity dispersion (GVD) coefficient, and $\gamma=2 \pi n_{2} / \lambda$ the nonlinear parameter with $n_{2}$ the nonlinear index and $\lambda$ the input laser wavelength. The first term of the right-hand side of Eq. (1) leads to the self- and cross-phase modulation (SPM and XPM) as well as four-wave mixing (FWM) processes. The second term accounts for the delayed Raman response through the $R_{R}(t)$ function [24]. Let us recall that, in $\mathrm{CS}_{2}$, the Raman frequency shift is $20 \mathrm{THz}$ and the gain linewidth equals $15 \mathrm{GHz}$ [25]. It is well known that the imaginary part of the Raman response in the Fourier domain is associated with the Raman gain, whereas the real part nonlinearly acts on the phase of the field. As both parts are closely linked by the Kramers-Kronig relations, the real part can reach significant values only around the Raman Stokes and antiStokes frequencies, leading to a strongly dispersive nonlinear index. The group index $n_{g}$ for the total field, given by the relation $n_{g}=n_{p}+\omega \frac{\partial n_{p}}{\partial \omega}$, presents an increase (decrease) at the Raman Stokes (anti-Stokes) frequency, all the larger as the phonon spectral resonance narrows. Correspondingly, the Raman Stokes component exhibits a strong decrease of its group velocity compared to that of the pump. This behavior characterizes the slow-light phenomenon.

We performed numerical simulations of Eq. (1) by assuming an input pulsed pump beam with a pulse duration of $80 \mathrm{ps}$ (FWHM) and a transverse Gaussian profile width of $52 \mu \mathrm{m}$ (FWHM). To initiate the SRS process, we add an input white noise level as a seed corresponding to the vacuum field fluctuations, that is, one photon per spatiotemporal mode. The input pump intensity $P_{i}=$ $0.8 \mathrm{GW} / \mathrm{cm}^{2}$ is chosen larger than the fundamental Kerr soliton intensity $P_{s}=0.25 \mathrm{GW} / \mathrm{cm}^{2}$ for which the spatial width of the pump beam at the waveguide output is equal to the input width.

Figures 1(a) and 1(b) illustrate (in gray scale) the longitudinal evolution of the space-integrated temporal intensity profiles for the pump and the Raman Stokes pulse, respectively. For the sake of clarity, these numerical data are normalized and therefore do not reveal the exponential growth of the Raman Stokes pulse. Nevertheless, it is clear from Fig. 1(a) that the pump pulse shape remains unchanged up to $1.7 \mathrm{~cm}$ of propagation (dashed white line) where depletion of its trailing edge occurs. On the other hand, we can see that the Raman pulse shown in Fig. 1(b) is first generated from noise at the center of the pump pulse with a shorter duration than the pump because of the Raman gain. Interestingly, it then undergoes a large optical delay (tens of ps) to the trailing edge of the pump while one may expect an opposite delay owing to the normal GVD regime $\left(\beta_{2}>0\right)$ at the pump wavelength. This optical delay is intimately related to the sharp group-velocity variation caused by the slow-light phenomenon. To verify this assertion, we have checked, by withdrawing the real part of the Raman response in our numerical tool, so that a weak opposite delay ( $\sim-3 \mathrm{ps}$ ) due to GVD appears instead. In addition, Fig. 1(b) shows that the optical delay due to the slow-light process stops at about $+25 \mathrm{ps}$ at the rear of the pump pulse because the Raman gain has vanished. This is fully consistent with the depletion in the trailing edge of the pump [seen in Fig. 1(a)].

It is interesting to note that, as the Raman pulse is slowed down during its amplification, it is also compressed. We observe the decrease of the Raman pulse duration that evolves from $34 \mathrm{ps}$ at the beginning to $28.5 \mathrm{ps}$ after $1.7 \mathrm{~cm}$ of propagation, i.e., at its maximum 25-ps delay. The fractional delay is thus equal to $25 / 28.5=0.88$. This value is comparable to that obtained by Sharping et al. [8]

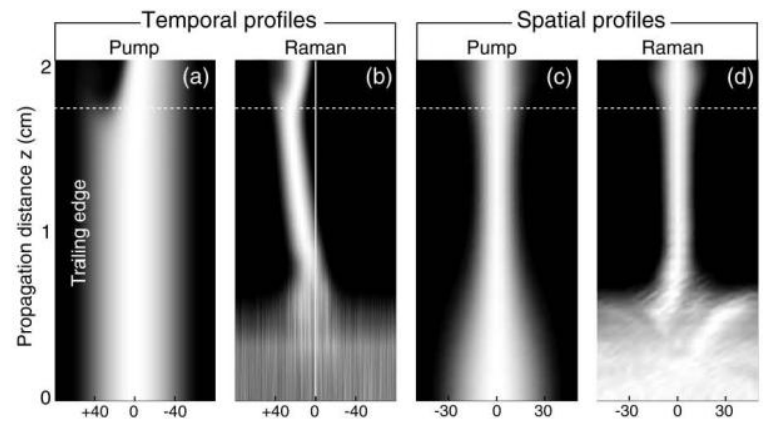

FIG. 1. Normalized temporal (a), (b) and spatial (c), (d) profiles (gray scale) for the (a), (c) pump pulse and (b), (d) the first Raman stokes order pulse during propagation along a $2 \mathrm{~cm}$ nonlinear planar waveguide. Input pump parameters: $80 \mathrm{ps}$ duration, input intensity $P_{i}=0.8 \mathrm{GW} / \mathrm{cm}^{2}$ (soliton power $P_{s}=0.25 \mathrm{GW} / \mathrm{cm}^{2}$ ). Other parameters are $\beta_{2}=+4.24 \times$ $10^{-25} \mathrm{~s}^{2} / \mathrm{m}, n_{2}=+3.5 \times 10^{-18} \mathrm{~m}^{2} / \mathrm{W}$, and $\lambda=532 \mathrm{~nm}$. 
in a Raman fiber amplifier with a 1-km highly nonlinear fiber (HNLF).

Regarding the spatial dynamics, Figs. 1(c) and 1(d) show the longitudinal evolution of the time-integrated spatial profiles of the pump and the Raman Stokes beam, respectively. First, it is clear from Fig. 1(c) that the pump beam undergoes a strong self-focusing in the first part of the waveguide, the input power being greater than the soliton power. It therefore tends to behave as a secondorder spatial soliton and then after $1.7 \mathrm{~cm}$ propagation (dashed line) spreads because of the Raman-induced depletion. Additionally, we see in Fig. 1(d) that the Raman Stokes beam, initially generated from noise, is spatially confined in the self-focused pump beam due to the Raman gain and XPM-induced mutual guiding [26]. It is significant that the Raman beam continues to propagate with a stable spatial width and behaves as a spatial soliton until the depletion of the pump. Note also that the Raman beam has a spatial width always smaller or equal than the pump.

To get a better insight, Figs. 2(a)-2(c) show the spatiotemporal intensity profiles for the pump at the waveguide's input and for the pump and the Raman Stokes components after $1.7 \mathrm{~cm}$ of propagation (i.e., before the pump depletion), respectively. The bottom insets represent the corresponding time-integrated spatial profiles. The direct comparison between Fig. 2(a) and 2(b) highlights both the strong self-focusing of the pump beam and the beginning of the depletion of its trailing edge. The bottom inset of Fig. 2(b) shows a weak spatial pedestal mainly due to the initiation of a second-order spatial soliton generation. As can be seen in Fig. 2(c), the Raman pulse is clearly observable in the trailing edge of the pump pulse due to the slow-light effect. More importantly, the bottom of Fig. 2(c) shows that the spatial profile of the Raman component has

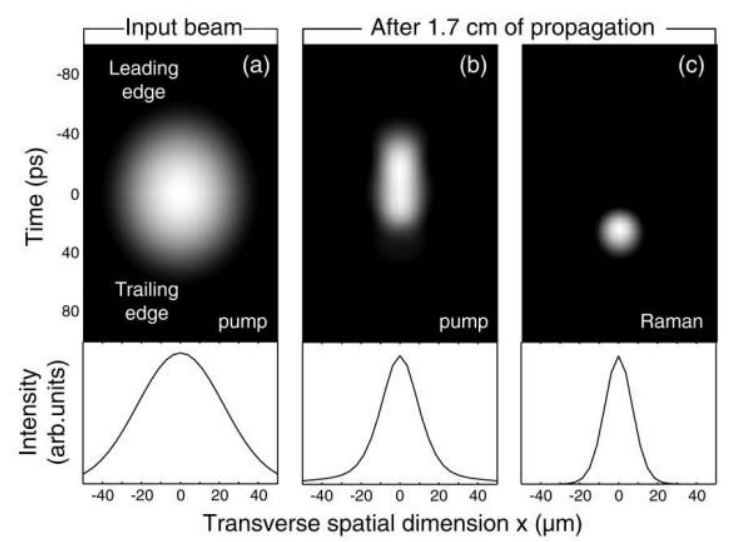

FIG. 2. Numerical spatiotemporal profiles of (a) the waveguide input pump and (b), (c) the pump and the first Raman Stokes order, respectively, at the beginning of the pump depletion regime for a $1.7 \mathrm{~cm}$ propagation length corresponding to the horizontal dashed line in Fig. 1. Bottom insets: corresponding time-integrated spatial profiles. Same input pump parameters as for Fig. 1. a clear-cut hyperbolic secant squared shape without any spatial pedestal, because it is only generated when the pump pulse attains a solitonlike regime and does not suffer from nonsolitonic parts.

The experimental setup is made up of a $3 \mathrm{~cm}$ long Kerr planar waveguide that consists of a $15-\mu \mathrm{m}$-thick liquid $\mathrm{CS}_{2}$ layer sandwiched between two SK 5 glass blocks (see Ref. [26] for more details). The pump source is a frequency-doubled $Q$-switched Nd:YAG powerchip laser at $532 \mathrm{~nm}$ with a $1 \mathrm{kHz}$ repetition rate and $370 \mathrm{ps}$ pulse duration. The input Gaussian beam is linearly polarized parallel to the TE mode of the waveguide. Its spatial width has been measured to be $52 \mu \mathrm{m}$. The pump and Raman components are spatially separated at the waveguide's output by using a diffraction grating (2400 lines $/ \mathrm{mm}$ ) and analyzed both in time and in space by the use of a Streak camera with a resolution of 5 ps.

Figures 3(a)-3(c) show the spatiotemporal experimental profiles recorded at the waveguide's output. Figure 3(a) is the pump in the diffraction regime, Fig. 3(b) the pump at the beginning of the depletion regime, and Fig. 3(c) the delayed Raman component, respectively. First, by comparing Fig. 3(a) and 3(b), it is clear that pump depletion occurs at the trailing edge in agreement with the position of the Raman Stokes order shown in Fig. 3(c), as predicted by our numerical simulations (see Fig. 1 and 2). The insets at the bottom represent the time-integrated spatial profiles fitted by the theoretical ones (dashed lines). It is important to note that the pump and the Raman beam both exhibit a spatial soliton profile. The Raman pulse, which is generated and slowed down by the SRS process, forms a slowlight spatial soliton.

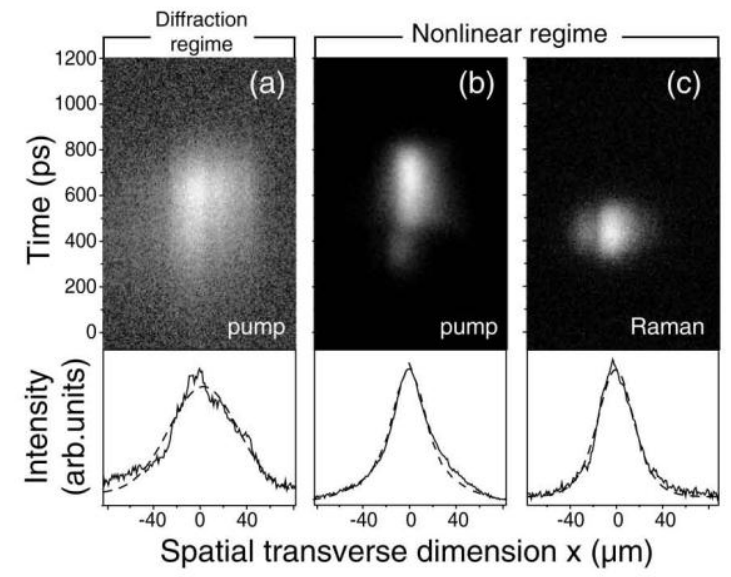

FIG. 3. Experimental spatiotemporal measurements at the waveguide output. (a) The pump pulse with a low input power $(1 \mathrm{~mW}),(\mathrm{b})$ the pump pulse at high input power $(1.74 \mathrm{~mW})$ at the beginning of the trailing edge depletion, and (c) the corresponding first Raman Stokes order pulse. Bottom insets: timeintegrated spatial profiles (straight curves) fitted by numerical profiles (dashed curves). 


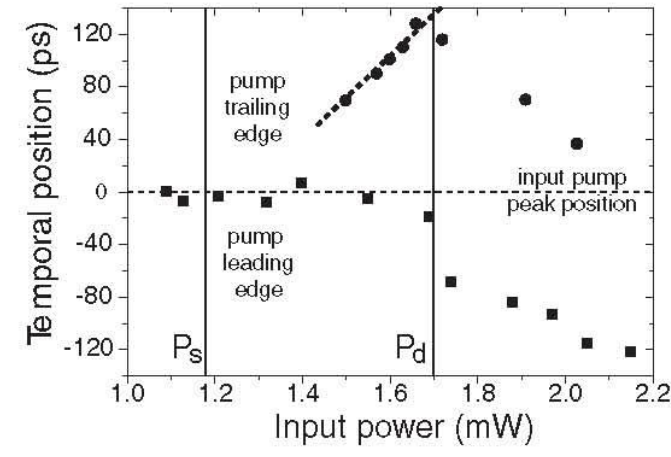

FIG. 4. Temporal position of the pump ( $\mathbf{\square}$ ) and the Raman pulse peaks at the waveguide's output in function of the mean input power. $P_{s}$ corresponds to the fundamental pump soliton power and $P_{d}$ corresponds to the power of the pump depletion.

Note that the slow-light process presented here has to be distinguished from the mechanism of group-velocity locking for the stimulated Raman $X$ waves generation in ultrashort optical pulse filamention though the observations seem to be similar to those reported here [27].

To analyze the slowing down of the soliton, we plot in Fig. 4 the output temporal positions of the pump and Raman pulse peaks in function of the mean input power. The difference between these two temporal positions represents the optical delay. For an input power lower than $1.7 \mathrm{~mW}$, i.e., before depletion of the pump trailing edge, the optical delay linearly depends on the input power, in good agreement with the theory of slow light [10]. The magnitude of the delay can then be tuned continuously to as much as $120 \mathrm{ps}$ by adjusting the pump power, leading to a minimum group velocity equal to $c / 2.8$. For the maximum time delay, the corresponding Raman pulse duration is equal to about $140 \mathrm{ps}$. The corresponding fractional delay of $120 / 140=0.85$ is in excellent agreement with our numerical predictions.

At higher input power, i.e., after the pump trailing edge depletion, the Raman pulse is generated where the pump is not still depleted, as numerically observed in Fig. 1(b). Then the Raman pulse moves forward to the leading edge of the pump during propagation, leading at the same time to the decrease of the pump pulse duration and also to the temporal shift of the pump peak toward the leading edge, as seen in Fig. 1(a) and 4.

To conclude, we have demonstrated both numerically and experimentally the phenomenon of self-guided slow light, i.e., a slow-light spatial Kerr soliton. This was achieved in a $\mathrm{CS}_{2}$ Kerr planar waveguide thanks to a sharp Raman gain band and a strong group-velocity dispersion at the Stokes frequencies. The Raman Stokes component was optically delayed by 120 ps for only a $3-\mathrm{cm}$ propagation length while exhibiting a spatial solitonic profile. Note also that the slow-light process presented here is at the heart of the multicolor soliton generation previously reported in Ref. [26].

The authors would like to thank L. Furfaro and R. Giust for a technical assistance. This work has been supported by the ministère délégué à la recherche.

[1] R.W. Boyd and D. J. Gauthier, in Progress in Optics, edited by E. Wolf (Elsevier, New York, 2002), Vol. 43, Chap. 6, p. 495.

[2] L. V. Hau et al., Nature (London) 397, 594 (1999).

[3] M. M. Kash et al., Phys. Rev. Lett. 82, 5229 (1999).

[4] A. V. Turukhin et al., Phys. Rev. Lett. 88, 023602 (2001).

[5] M.S. Bigelow, N. N. Lepeshkin, and R.W. Boyd, Phys. Rev. Lett. 90, 113903 (2003); Science 301, 200 (2003).

[6] K. Y. Song et al., Opt. Express 13, 82 (2005).

[7] Y. Okawachi et al., Phys. Rev. Lett. 94, 153902 (2005).

[8] J.E. Sharping et al., Opt. Express 13, 6092 (2005).

[9] F. L. Kien et al., IEEE J. Sel. Top. Quantum Electron. 9, 93 (2003).

[10] K. Lee and N. M. Lawandy, Appl. Phys. Lett. 78, 703 (2001).

[11] B. Pesala et al., Opt. Express 14, 12968 (2006).

[12] M. Scalora et al., Phys. Rev. E 54, R1078 (1996).

[13] M. L. Povinelli et al., Opt. Express 13, 7145 (2005).

[14] J. F. McMillan et al., Opt. Lett. 31, 1235 (2006).

[15] U. Leonhardt, arXiv:quant-ph/0408046.

[16] A. V. Rybin et al., Phys. Rev. E 72, 026613 (2005).

[17] J. Leon, Phys. Rev. A 75, 063811 (2007).

[18] D. N. Christodoulides and R. I. Joseph, Phys. Rev. Lett. 62, 1746 (1989)

[19] A.B. Aceves and S. Wabnitz, Phys. Lett. A 141, 37 (1989).

[20] B. J. Eggleton et al., J. Opt. Soc. Am. B 16, 587 (1999).

[21] J. T. Mok et al., Nature Phys. 2, 775 (2006).

[22] A.A. Sukhorukov and Y. S. Kivshar, Phys. Rev. Lett. 97, 233901 (2006).

[23] G. I. Stegeman and M. Segev, Science 286, 1518 (1999).

[24] G. P. Agrawal, Nonlinear Fiber Optics (Academic, New York, 2001), 3rd ed.

[25] R.W. Boyd, in Nonlinear Optics (Academic, New York, 1992).

[26] G. Fanjoux et al., Opt. Lett. 31, 3480 (2006).

[27] D. Faccio et al., Opt. Lett. 32, 184 (2007). 